\title{
Evolving challenges and opportunities for difficult airway management guidelines
}

\author{
Sylvain Boet, MD • M. Dylan Bould, MB ChB • \\ Pierre Diemunsch, MD, PhD
}

Published online: 1 June 2011

(C) Canadian Anesthesiologists' Society 2011

\begin{abstract}
Airway management is a key competency for every anesthesiologist. In 1990, Caplan et al. reported that 34\% of anesthesia-related claims were related to airway management. ${ }^{1}$ Since that time, several national airway management guidelines have been published with the aim to establish and promote safer airway management practice. ${ }^{2,3}$ Since the publication of those guidelines, studies have shown a decrease in anesthesia complications related to airway management. ${ }^{4,5}$ Of course, it is not possible to determine causality, and there have been other significant changes in practice over this time, e.g., supraglottic airways are now used in more than half the patients in the United Kingdom (UK). ${ }^{5}$ Unfortunately, despite these advances in both protocols and technology, airway complications in anesthesia still occur, and they are often associated with severe complications, i.e., brain damage or death. ${ }^{5}$ Herein, we consider the contents of future airway management guidelines that might create a further impact on patient safety, while bearing
\end{abstract}

Attributable to: Department of Anesthesiology, St. Michael's Hospital, University of Toronto, 30 Bond St., Toronto, ON M5B 1W8, Canada.

S. Boet, MD ( $\varangle)$

Department of Anesthesiology, Saint Michael's Hospital, University of Toronto, 30 Bond Street, Toronto, ON M5B 1W8, Canada

M. D. Bould, MB ChB

The Children's Hospital of Eastern Ontario,

University of Ottawa, Ottawa, ON, Canada

P. Diemunsch, MD, PhD

Department of Anaesthesiology, Critical Care and Prehospital Emergency Medicine, Hôpital de Hautepierre,

University of Strasbourg, Strasbourg, France in mind the known problematic adherence to airway management guidelines, the role of medical education, and the rapid evolution of new airway management technology and skills.

When we consider the history of major developments in airway management, we observe a recent acceleration of the development of technology and skills in this area. The Macintosh and Miller blades, designed in the 1940s when curare was introduced into anesthesia, are still in common use. Major incremental steps in airway management occurred with the development of fibreoptic intubation in the early 1970s, and again in the 1980s, with the invention of the laryngeal mask airway $\left(\mathrm{LMA}^{\mathrm{TM}}\right.$ ) and the rigid fibreoptic laryngoscopes (e.g., Bullard ${ }^{\mathrm{TM}}$ ). Other major advances were made in the 1990s with the intubating laryngeal mask airway (ILMA), devices for transillumination (e.g., Trachlight ${ }^{\mathrm{TM}}$ ), and videolaryngoscopy. During the last decade, anesthesiologists have used combinations of these new techniques to produce even further airway management possibilities, e.g., the combination of the LMA or the ILMA with fibreoptic bronchoscopy. The recent availability of sugammadex may change airway management practice and should be considered in new airway management guidelines. In addition to these "quantum leaps", there has also been a rapid increase in the number of new versions of very similar airway management devices, ${ }^{6}$ in part because of the "me too device" phenomenon. This increase in technology provides both advantages and disadvantages to anesthesiologists. Although we have more options for managing difficult intubation, it can be difficult to know the appropriate airway tool to use for a given purpose, especially considering the logistic challenges of conducting controlled trials on patients with difficult airways. A key tension for future guidelines is to reconcile the increasing number of new airway management options with 
the knowledge that simple guidelines are more likely to be remembered and maintained.

The first airway management guidelines were published in $1993^{3,7}$ a relatively recent development in the history of airway management. Despite mini-revolutions in airway management, guidelines have lagged behind; even the most recent guidelines published within the past few years do not mention many of the new airway devices used widely in everyday practice. The pace of development of new skills and technology suggests the need for more frequent reviews or revisions of guidelines; however, airway management technology develops faster than the laborious process of elaborating consensus guidelines. Practice guidelines have to be up-to-date for clinicians to apply them effectively. Then again, Shekelle et al. concluded that the average lifespan of guidelines in general is very limited, and most are outdated after three to six years. ${ }^{8}$ There is no need to revise airway guidelines after the invention of each new airway tool, but they certainly should reflect significant developments, such as videolaryngoscopy.

Other difficult questions arise as some new devices and techniques push the boundaries of the definition of difficult intubation to a point where the definition of difficult intubation itself may need to be reconsidered. ${ }^{9}$ For example, videolaryngoscopy is designed to offer the advantage of rapid visualization of the glottis on an external screen without having to align oral, pharyngeal, and tracheal axes. This technique results in improved laryngeal views compared with direct laryngoscopy ${ }^{10}$ and achieves an improved success rate of successful tracheal intubations. ${ }^{9}$ At present, the technique is used increasingly in everyday practice for patients with both predicted easy and predicted difficult tracheal intubations with a Macintosh blade. Our limited understanding of how to predict difficult intubation is obscured further by the lack of data on predicting difficult intubation with newer techniques. The literature on intubation is based on laryngoscopy with a Macintosh blade, and prediction of difficult videolaryngoscopy has rarely been studied. ${ }^{11}$ Direct laryngoscopy with a Macintosh blade is an inexpensive technique, and the procedural skill is well retained when learned to a mastery level. However, we should consider whether we should continue teaching the use of the Macintosh laryngoscope as the first-line tool for intubation, particularly for specialties, such as emergency medicine and intensive care, where intubation is a relatively infrequent event and difficult intubation tends to be associated with worse outcomes. ${ }^{12}$ Indeed, several studies have shown that videolaryngoscopy makes intubation easier for both anesthesiologists and non-anesthesiologists ${ }^{6,9,13}$ and in patients with both normal and difficult intubation. ${ }^{14}$

It has been demonstrated that there is a poor adherence to guidelines by anesthesiologists both in Europe and in North America. ${ }^{4,15}$ Data from two nationwide analyses, including a recent year-long prospective study in the UK with a denominator of 2.9 million anesthetics, have shown that deviations from standard practice, human factors, nontechnical skills, and training opportunities play a clear role in airway complications and mortality in anesthesiology. ${ }^{4,5}$ Therefore, a key challenge for future airway management guidelines will be to integrate evidence-based education within evidence-based medicine. Even for experienced anesthesiologists, complex guidelines are challenging to implement in practice. ${ }^{15}$ Guidelines are developed to reach the best compromise between the most complex evidencebased treatments and realistic implementation in real life, with emphasis on skills that are most easily learned and retained. Despite calls almost 15 years ago to incorporate training into guidelines, ${ }^{16}$ most of the current airway guidelines have neglected to implement an educational plan (including the teaching of vital human factors skills) into their recommendations. In 2005 and 2010, resuscitation guidelines included an increasing focus on education and human factors for teaching Advanced Cardiac Life Support and optimizing its implementation. ${ }^{17}$ Hence, it might be beneficial to consider a clear education strategy to promote implementation of airway guidelines in a clinical setting and to include elements of team training and human factors. ${ }^{17}$ More educational research is needed, addressing the following key issues: the different learning curves involved with various techniques; current variations in skill level amongst anesthesiologists; the use of simulation-based medical education; non-technical skills, such as situation awareness and resource management; the effect of stress on performance; and factors that affect the long-term retention of skills and knowledge for uncommonly occurring events. Interdisciplinary collaboration between medicine and education specialists may help to ensure that new airway management guidelines actually impact on patient safety.

Many countries have published their national airway management guidelines. ${ }^{2,3}$ Unlike well-accepted international guidelines for neonatal, pediatric, and adult resuscitation, there have been no international airway management guidelines. Instead, individual organizations must repeat the resource intensive process of developing consensus guidelines and, in a way, "reinvent the wheel". The need to revise guidelines frequently likely contributes to the challenge faced by every organization. Although the idea of international consensus guidelines is appealing, this is one area where airway management guidelines would differ significantly from resuscitation guidelines. There is a significant variation in resources and skill levels of healthcare providers in various countries, institutions, and even departments within institutions, e.g., anesthesia, intensive care, and emergency departments. ${ }^{12}$ While a two- to threeday course in Advanced Cardiac Life Support is effective in teaching resuscitation skills to a wide range of healthcare professionals, this is not the case with advanced airway 
management where certain technical skills require long periods of practice.

In order to standardize future guidelines, a group of major journals in anesthesia and perioperative medicine, including the Canadian Journal of Anesthesia, have recently taken action to improve new published practice guidelines, not only for the airway but for guidance in clinical care in general. ${ }^{18}$ Among these new requirements, future guidelines on a particular topic must refer to information contained in previous guidelines and give details concerning the clinical problem to be tackled, the basis for generating the statement, a review of the evidence for the statement, and the statement itself. The new requirements aim to produce well-structured guidelines with clear explanations to facilitate clinicians' proficiency in implementing the procedures. To minimize confusion and to enhance transparency, the new version of the "Instructions for Authors" should specify how each new guideline corresponds with the numerous existing guidelines published by other societies and organizations.

In conclusion, recent "quantum leaps" in airway management should prompt review of current airway management guidelines. Revised recommendations will need to overcome several key tensions, including reconciliation of the increasing number of new airway management options with the laborious process of consensus guidelines; integration of evidence-based education within evidence-based medicine; and possible handling of the current lack of international guidelines. The resolution of these main conflicts may further improve patient safety. Innovative classifications or guideline structuring may need to be considered in future guidelines to benefit from the advances in technology without losing clarity to achieve the ultimate aim of improving patient safety.

\section{Les directives de prise en charge des voies aériennes difficiles: évolution des défis et des possibilités}

La prise en charge des voies aériennes est une compétence clé de tout anesthésiologiste. En 1990, Caplan et coll. ont rapporté que $34 \%$ des poursuites légales en rapport avec l'anesthésie étaient liées à la prise en charge des voies aériennes. ${ }^{1}$ Depuis, de nombreuses directives nationales ont été publiées à ce sujet, avec pour objectif d'établir et de promouvoir une pratique de prise en charge des voies aériennes plus sécuritaire. ${ }^{2,3}$ Depuis la publication de ces directives, des études montrent une réduction des complications anesthésiques liées à la prise en charge des voies aériennes. ${ }^{4,5}$ Bien entendu, il est impossible d'établir ici un lien de causalité; en outre, d'autres changements importants au niveau de la pratique ont également eu lieu simultanément, notamment l'utilisation des voies aériennes supraglottiques chez plus de la moitié des patients au Royaume-Uni (R.-U.). ${ }^{5}$ Malheureusement, malgré les progrès accomplis au niveau des protocoles et de la technologie, les complications au niveau des voies aériennes en anesthésie continuent de survenir, et elles sont souvent associées à des complications graves, notamment à des lésions cérébrales et des décès. Dans cet éditorial, nous réfléchissons au contenu futur des directives de prise en charge des voies aériennes, qui pourraient avoir un impact encore plus important sur la sécurité des patients, tout en gardant à l'esprit le problème bien connu du respect de telles directives, le rôle de la formation médicale ainsi que l'évolution rapide des nouvelles technologies et compétences de prise en charge des voies aériennes.

Lorsqu'on repense aux principaux développements survenus dans la prise en charge des voies aériennes, on observe une accélération récente du développement des technologies et des compétences dans ce domaine. Les lames Macintosh et Miller, conçues dans les années 1940 lors de l'introduction des curares en anesthésie, sont encore couramment utilisées. Plusieurs progrès majeurs dans la prise en charge des voies aériennes ont pu voir le jour grâce à la mise au point de l'intubation par fibre optique au début des années 1970 puis, au cours des années 1980, à l'invention du masque laryngé $\left(\mathrm{LMA}^{\mathrm{TM}}\right)$ et des laryngoscopes rigides à fibre optique (par ex., Bullard ${ }^{\mathrm{TM}}$ ). Dans les années 1990, il y a eu d'autres progrès importants, notamment le masque laryngé d'intubation (ILMA), les dispositifs pour la transillumination ( $p a r$ ex. Trachlight ${ }^{\mathrm{TM}}$ ) et la vidéolaryngoscopie. Au cours des dix dernières années, les anesthésiologistes ont combiné ces nouvelles techniques afin de disposer d'encore plus de possibilités de prise en charge des voies aériennes, en combinant par exemple le LMA ou l'ILMA avec la bronchoscopie par fibre optique. L'addition récente du sugammadex à l'arsenal thérapeutique pourrait changer la pratique de prise en charge des voies aériennes; un tel développement devrait être traité dans les nouvelles directives de prise en charge des voies aériennes. Outre ces bonds prodigieux, nous avons également assisté à une augmentation rapide du nombre de nouvelles versions de dispositifs très similaires de prise en charge des voies aériennes ${ }^{6}$ - en partie en raison du phénomène du 'moi aussi j'en veux un'. Cette augmentation des technologies est accompagnée à la fois d'avantages et d'inconvénients pour les anesthésiologistes. Bien que nous disposions d'un nombre plus important d'options pour la prise en charge d'une intubation difficile, il peut être difficile de savoir quel outil utiliser à quelle fin, particulièrement lorsqu'on pense 
aux défis logistiques survenant lorsqu'on souhaite réaliser des études contrôlées chez des patients présentant des voies aériennes difficiles. L'un des objectifs essentiels à atteindre dans les prochaines directives sera de concilier le nombre croissant de nouvelles options de prise en charge des voies aériennes au fait que des directives simples sont plus faciles à garder à l'esprit et à respecter.

Les premières directives sur la prise en charge des voies aériennes ont été publiées en $1993^{3,7}$ un progrès relativement récent dans l'histoire de la prise en charge des voies aériennes. Malgré les mini-révolutions auxquelles on a assisté dans le domaine, les directives sont restées à la traîne; en effet, même les directives les plus récentes publiées ces dernières années ne mentionnent pas certains nouveaux dispositifs de prise en charge des voies aériennes couramment utilisés dans la pratique quotidienne. En raison de la vitesse de développement des nouvelles compétences et des nouvelles technologies, des révisions plus fréquentes des directives sont nécessaires; toutefois, les technologies de prise en charge des voies aériennes sont plus rapidement mises au point que le processus laborieux nécessaire à l'élaboration de directives consensuelles. Si l'on veut que les cliniciens les appliquent de façon efficace, les directives de pratique doivent être à jour. En revanche, Shekelle et coll. ont conclu que la durée de vie moyenne de directives est, en règle générale, très limitée, et que la plupart deviennent obsolètes après trois à six ans. ${ }^{8}$ Il n'est pas nécessaire de réviser les directives sur la prise en charge des voies aériennes à chaque lancement d'un nouveau dispositif, mais elles devraient indubitablement aborder les principaux développements, comme la vidéolaryngoscopie.

D'autres questions difficiles surgissent alors que de nouveaux dispositifs et de nouvelles techniques repoussent les limites de ce qu'on entend par intubation difficile, à tel point que la définition même d'une intubation difficile pourrait devoir être repensée. ${ }^{9}$ Par exemple, la vidéolaryngoscopie est conçue de façon à offrir l'avantage d'une visualisation rapide de la glotte sur un écran externe, sans avoir à aligner les axes oral, pharyngé et trachéal. Grâce à cette technique, on dispose de meilleures images laryngées par rapport à la laryngoscopie directe, ${ }^{10}$ ce qui contribue à un taux de réussite plus élevé des intubations trachéales. ${ }^{9}$ À l'heure actuelle, cette technique est de plus en plus utilisée dans la pratique quotidienne chez les patients dont l'intubation trachéale est anticipée comme facile ou difficile avec une lame de Macintosh. Notre compréhension limitée des façons d'anticiper une intubation difficile est encore plus compliquée en raison de l'absence de données traitant de l'identification précoce d'une intubation difficile avec les techniques récentes. $\mathrm{La}$ littérature portant sur l'intubation se fonde sur une laryngoscopie avec une lame de Macintosh, et la prédiction d'une vidéolaryngoscopie difficile n'a été que très peu étudiée. ${ }^{11}$ La laryngoscopie directe à l'aide d'une lame de Macintosh est une technique dispendieuse, et les compétences pour réaliser cette intervention ne sont bien assimilées que lorsqu'elles sont véritablement maitrisées à un haut niveau. Toutefois, il convient de bien réfléchir afin de déterminer si nous devrions continuer à enseigner l'utilisation d'un laryngoscope Macintosh en tant que dispositif d'intubation de première ligne, particulièrement dans les spécialités telles que la médecine d'urgence et les soins intensifs, où l'intubation est relativement rare et où l'intubation difficile a tendance à être associée à des devenirs moins bons. ${ }^{12}$ En effet, plusieurs études ont montré que la vidéolaryngoscopie facilite l'intubation, tant pour les anesthésiologistes que pour les non-anesthésiologistes, ${ }^{6,9,13}$ que l'intubation soit facile ou difficile. ${ }^{14}$

Il a été démontré que les anesthésiologistes respectent peu les directives, aussi bien en Europe qu'en Amérique du Nord. ${ }^{4,15}$ Des données tirées de deux analyses à l'échelle nationale, notamment une récente étude prospective d'un an réalisée au R.-U. avec un dénominateur de 2,9 millions d'anesthésies, ont montré que les écarts par rapport à la pratique normalisée, les facteurs humains, les compétences non techniques et les occasions de formation jouent un rôle important dans les complications au niveau des voies aériennes et dans la mortalité en anesthésiologie. ${ }^{4,5}$ C'est pourquoi l'un des principaux défis des directives futures de prise en charge des voies aériennes sera d'intégrer une formation fondée sur des données probantes dans une médecine fondée sur des données probantes. Même pour un anesthésiologiste aguerri, des directives compliquées sont difficiles à mettre en œuvre dans la pratique. ${ }^{15}$ L'objectif de directives, quelles qu'elles soient, est de parvenir au meilleur compromis possible entre les traitements fondés sur des données probantes les plus complexes et une mise en œuvre réaliste dans la vraie vie, avec une emphase sur les compétences les plus faciles à acquérir et à retenir. Malgré plusieurs appels, il y a près de 15 ans, à l'intégration de la formation dans les directives, ${ }^{16}$ la plupart des directives actuelles traitant de la prise en charge des voies aériennes n'ont pas mis en œuvre de plan de formation (y compris l'enseignement des compétences vitales de facteurs humains) dans leurs recommandations. En 2005 et 2010, les directives de réanimation ont mis une emphase particulière sur la formation et les facteurs humains dans l'enseignement de la réanimation cardiorespiratoire avancée (Advanced Cardiac Life Support - ACLS) et sur la promotion de sa mise en œuvre. ${ }^{17}$ Ainsi, il pourrait être intéressant d'envisager une stratégie de formation claire afin de promouvoir la mise en œuvre des directives de prise en charge des voies aériennes dans un cadre clinique et d'inclure des éléments de formation en équipe ainsi que des facteurs humains. ${ }^{17}$ Des recherches 
plus poussées sur la formation sont nécessaires; ces recherches devraient aborder les questions clés suivantes : les différentes courbes d'apprentissage selon les différentes techniques; les variations actuelles dans le niveau de compétence des anesthésiologistes; l'utilisation d'une formation médicale fondée sur la simulation; les compétences non techniques, comme la prise de conscience de la situation et la gestion des ressources; l'effet du stress sur les performances; et les facteurs qui affectent la rétention à long terme des compétences et des connaissances concernant les événements peu courants. En promouvant une collaboration interdisciplinaire entre les spécialistes de la médecine et de la formation, on pourrait contribuer à garantir que les nouvelles directives concernant la prise en charge des voies aériennes ont un impact réel sur la sécurité des patients.

De nombreux pays ont publié des directives nationales de prise en charge des voies aériennes. ${ }^{2,3}$ Au contraire des directives internationales bien établies pour la réanimation chez le nouveau-né, l'enfant et l'adulte, il n'existe pas de directives internationales pour la prise en charge des voies aériennes. Au lieu de cela, plusieurs organismes travaillant chacun de leur côté doivent répéter le processus de mise au point de directives consensuelles, un processus qui accapare de nombreuses ressources, et, d'une certaine manière, qui «réinvente la roue ». La nécessité de révisions fréquentes des directives contribue probablement à rendre la tâche encore plus ardue pour chaque organisme. Bien que l'idée de directives consensuelles internationales soit séduisante, la prise en charge des voies aériennes est très différente de la réanimation en ce qui touche à l'élaboration de directives communes. En effet, on observe d'importantes différences en termes de ressources et de niveaux de compétences des fournisseurs de soins de santé d'un pays à l'autre, d'une institution à une autre, et même d'un département à un autre au sein d'une même institution, par exemple entre les départements d'anesthésie, de soins intensifs et de médecine d'urgence. ${ }^{12}$ Bien qu'une formation de deux à trois jours en ACLS soit efficace pour enseigner les compétences nécessaires à la réanimation à un vaste éventail de professionnels de la santé, ce n'est pas le cas des techniques de prise en charge avancée des voies aériennes, dont certaines requièrent davantage de pratique.

Afin de normaliser les directives futures, un groupe incluant les principales revues d'anesthésie et de médecine périopératoire, y compris le Journal canadien d'anesthésie, a récemment décidé d'agir dans le but d'améliorer les nouvelles directives publiées - non seulement en matière de prise en charge des voies aériennes, mais aussi pour améliorer les soins cliniques en général. ${ }^{18}$ Parmi les nouvelles exigences, les directives futures portant sur un sujet en particulier devront renvoyer à des informations contenues dans les directives précédentes et donner des détails concernant le problème clinique à aborder, le raisonnement derrière l'énoncé, une révision des données probantes soutenant l'énoncé, et l'énoncé lui-même. Les nouvelles exigences ont pour but la création de directives bien structurées et qui offrent des explications claires; ainsi, les cliniciens pourront mieux mettre en œuvre les interventions nécessaires. Afin de minimiser la confusion et d'améliorer la transparence, la nouvelle version des «Directives aux auteurs » devra spécifier la façon dont chaque nouvelle directive correspond aux nombreuses directives existantes publiées par d'autres sociétés et organismes.

En conclusion, les bonds prodigieux accomplis récemment dans la prise en charge des voies aériennes devraient susciter la révision des directives actuelles de prise en charge des voies aériennes. Les recommandations révisées devront aller au-delà de plusieurs problèmes clés, notamment en réconciliant le nombre croissant de nouvelles options de prise en charge des voies aériennes au processus laborieux de création de directives consensuelles; en intégrant la formation fondée sur des données probantes à la médecine fondée sur des données probantes; et en remédiant, peut-être, à l'absence actuelle de directives internationales. La résolution de ces importants conflits pourrait améliorer encore davantage la sécurité des patients. Des classifications innovantes et la structuration des directives pourraient devoir être envisagées dans les directives futures afin de tirer profit des progrès technologiques, sans pour autant perdre en précision, et atteindre notre but ultime : améliorer la sécurité des patients.

Acknowledgement The authors sincerely thank Dr. Edward Crosby for his constructive review of an earlier version of this manuscript.

Conflict of interest None declared.

Financial support No financial support to declare.

\section{References}

1. Caplan RA, Posner KL, Ward RJ, Cheney FW. Adverse respiratory events in anesthesia: a closed claims analysis. Anesthesiology 1990; 72: 828-33.

2. American Society of Anesthesiologists Task Force on Management of the Difficult Airway. Practice guidelines for management of the difficult airway: an updated report by the American Society of Anesthesiologists Task Force on Management of the Difficult Airway. Anesthesiology 2003; 98: 1269-77.

3. Henderson JJ, Popat MT, Latto IP, Pearce AC. Difficult Airway Society guidelines for management of the unanticipated difficult intubation. Anaesthesia 2004; 59: 675-94.

4. Lienhart A, Auroy Y, Pequignot F, et al. Survey of anesthesiarelated mortality in France. Anesthesiology 2006; 105: 1087-97.

5. Cook TM, Woodall N, Frerk C; on behalf of the Fourth National Audit Project. Major complications of airway management in the 
UK: results of the Fourth National Audit Project of the Royal College of Anaesthetists and the Difficult Airway Society. Part 1: Anaesthesia. Br J Anaesth 2011; 106: 617-31.

6. Malik MA, O'Donoghue C, Carney J, Maharaj CH, Harte BH, Laffey JG. Comparison of the Glidescope, the Pentax AWS, and the Truview EVO2 with the Macintosh laryngoscope in experienced anaesthetists: a manikin study. Br J Anaesth 2009; 102: 128-34.

7. Anonymous. Practice guidelines for management of the difficult airway. A report by the American Society of Anesthesiologists Task Force on Management of the Difficult Airway. Anesthesiology 1993; 78: 597-602.

8. Shekelle PG, Ortiz E, Rhodes S, et al. Validity of the Agency for Healthcare Research and Quality clinical practice guidelines: how quickly do guidelines become outdated? JAMA 2001; 286: 1461-7.

9. van Zundert A, Maassen R, Lee R, et al. A Macintosh laryngoscope blade for videolaryngoscopy reduces stylet use in patients with normal airways. Anesth Analg 2009; 109: 825.

10. Sun DA, Warriner CB, Parsons DG, Klein R, Umedaly HS, Moult $M$. The GlideScope Video Laryngoscope: randomized clinical trial in 200 patients. Br J Anaesth 2005; 94: 381-4.

11. Yentis SM. Predicting difficult intubation-worthwhile exercise or pointless ritual? Anaesthesia 2002; 57: 105-9.

12. Cook TM, Woodall N, Harper J, Benger J; on behalf of the Fourth National Audit Project. Major complications of airway management in the UK: results of the Fourth National Audit Project of the Royal College of Anaesthetists and the Difficult Airway Society. Part 2: intensive care and emergency departments. Br J Anaesth 2011; 106: 632-42.

13. Howard-Quijano KJ, Huang YM, Matevosian R, Kaplan MB, Steadman $R H$. Video-assisted instruction improves the success rate for tracheal intubation by novices. Br J Anaesth 2008; 101: 568-72.

14. Enomoto Y, Asai T, Arai T, Kamishima K, Okuda Y. Pentax-AWS, a new videolaryngoscope, is more effective than the Macintosh laryngoscope for tracheal intubation in patients with restricted neck movements: a randomized comparative study. Br J Anaesth 2008; 100: 544-8.

15. Borges BC, Boet $S$, Siu $L W$, et al. Incomplete adherence to the ASA difficult airway algorithm is unchanged after a high-fidelity simulation session. Can J Anesth 2010; 57: 644-9.

16. Crosby ET, Cooper RM, Douglas MJ, et al. The unanticipated difficult airway with recommendations for management. Can J Anaesth 1998; 45: 757-76.

17. Soar J, Monsieurs KG, Ballance JH, et al. European Resuscitation Council Guidelines for Resuscitation 2010 Section 9. Principles of education in resuscitation. Resuscitation 2010; 81: 1434-44.

18. 2011 Editorial Board, Canadian Journal of Anesthesia. 2011 Canadian Journal of Anesthesia Guide for Authors. Can J Anesth 2011; DOI:10.1007/s12630-011-9516-4. 